\title{
The Use of Fukuyama's Sulfonamide in the Synthesis of Selectively Protected Spermidines
}

\author{
Emerson T. da Silva, Fátima S. Fona and Edson L. S. Lima* \\ Instituto de Química, Universidade Federal do Rio de Janeiro, Cidade Universitária, \\ 21945-970 Rio de Janeiro - RJ, Brazil
}

\begin{abstract}
A diferenciação dos grupos amino terminais de poliaminas freqüentemente envolve uma série de etapas de proteção e desproteção, resultando em rotas sintéticas longas e de baixo rendimento global. Tendo em vista o relevante papel biológico atribuído a estas substâncias, torna-se necessário o desenvolvimento de sínteses mais eficientes para poliaminas. Neste artigo descrevemos uma síntese de duas espermidinas seletivamente protegidas, empregando a sulfonamida de Fukuyama. As duas sínteses foram executadas em três etapas a partir da 1,3-propanodiamina, com um rendimento global superior a $40 \%$.
\end{abstract}

The differentiation of terminal amino groups in polyamines usually involves a series of protection and deprotection steps, leading to long reaction sequences with low overall yields. Given the relevance of the biological activities displayed by polyamines, the development of more efficent synthetic routes for these compounds is highly desired. Herein we report the synthesis of two selectively protected spermidines, using Fukuyama's sulfonamide. Both syntheses were performed in three steps, starting from 1,3-diaminopropane, with an overall yield higher than $40 \%$.

Keywords: polyamines, spermidine, Fukuyama's sulfonamide

\section{Introduction}

In the past decades a great deal of attention has been given to polyamines, especially because of their involvement in the regulation of cellular functions, such as cell proliferation and differentiation. ${ }^{1}$ Additionally, a wide range of biological activities have been attributed to polyamines conjugates and derivatives, such as antianigogenic, anticancer, and neurotoxins, to name a few. ${ }^{2}$

The metabolism of polyamines in prokaryotes has also gained increased importance. ${ }^{3}$ In fact, the inhibition of enzymes involved in the metabolism of parasitic protozoa has been recognized as a promising strategy for the chemotherapy of tropical diseases. ${ }^{4}$ For instance, the inhibition of ornithine descaboxylase by a-difluoromethylornithine, a drug candidate against African sleeping sickness and malaria, ${ }^{4,5}$ blocks the first step of polyamine biosynthetic pathway. ${ }^{6}$ Trypanothione, structurally characterized as $\mathrm{N}^{1}, \mathrm{~N}^{8}$-bis(glutathionyl)spermidine, is a polyamine derivative used by trypanosomatids as a defense against reactive oxygen species during their infective cycle. ${ }^{7}$ The metabolism of trypanothione is another target

* e-mail: edson@iq.ufrj.br for drug development against trypanosomiases and leishmanioses. ${ }^{8}$

As part of a research program aiming the synthesis of peptide-polyamine conjugates, we became interested in the preparation of selectively protected spermidines. ${ }^{9}$ However, the synthesis of unsymmetrical polyamines usually requires several protection and deprotection steps, making such approaches unnatractive due to the long reaction sequences involved..$^{10}$ More straighforward alternatives to acess polyamine backbones are needed.

The dual role of Fukuyama's sulfonamide, ${ }^{11}$ which not only masks the amino group, but also activates it to grow the polyamine chain, meet the requirements to solve those issues. ${ }^{12}$ Herein, we wish to describe a versatile route for two orthogonally protected spermidines using Fukuyama's sulfonamide.

\section{Results and Discussion}

The first synthesis starts with the monoprotection of 1,3-propanediamine with 2-nitrobenzenosulfonyl chloride (NBS-Cl) in 70\% yield, using a modification of the protocol described by Haemers. ${ }^{13,14}$ The remaining amino group was protected with 1,3-dimethyl-5-acetyl-barbituric acid (DAB) to give $\mathbf{1}$ in $70 \%$ yield. ${ }^{14}$ Selective N-alkylation 
with 4-bromobutylphthalimide using $\mathrm{K}_{2} \mathrm{CO}_{3}$ as base in refluxing acetonitrile afforded the protected spermidine $\mathbf{2}$, in good yield. ${ }^{15}$

Since both phthalimide and DAB groups of spermidine 2 can be removed with hydrazine, we decided to proof that under controlled conditions a selective deprotection may be achieved. ${ }^{14}$ While the removal of the phthalimide group is usually carried out in refluxing ethanol over $3-5 \mathrm{~h}$, the DAB group can be cleaved at $0{ }^{\circ} \mathrm{C}$. Thus, by treatting 2 with aqueous hydrazine in $\mathrm{THF}$ at $0{ }^{\circ} \mathrm{C}$, selective removal of DAB was performed, affording spermidine 3 in $82 \%$ yield.

A similar strategy was employed to prepare spermidine $\mathbf{5}$. Accordingly, the monoprotection of 1,3-propanediamine with (Boc) ${ }_{2} \mathrm{O}^{16}$ followed by reaction with $\mathrm{NBS}-\mathrm{Cl}$, afforded the known sulfonamide 4, in $62 \%$ yield (two steps). Reaction of 4 with 4-bromobutylphthalimide using $\mathrm{K}_{2} \mathrm{CO}_{3}$ in refluxing acetonitrile over $12 \mathrm{~h}$ provided the orthogonally protected spermidine 5, in $71 \%$ yield. End-group differentiation of a parent spermidine has already been demonstrated by us. ${ }^{9}$

In conclusion, our methodology provides an efficient alternative for the synthesis of orthogonally protected spermidines in a short sequence of steps with good overall yields, from inexpensive starting materials. The Fukuyama's sulfonamide can be introduced at different stages, and acts both as an activating group for $\mathrm{N}$-alkylation and as an orthogonal protective group. Additionally, depending on the temperature, DAB and phtalimide groups can show orthogonal behavior.<smiles>NCCCNS(=O)(=O)c1ccccc1[N+](=O)[O-]</smiles>

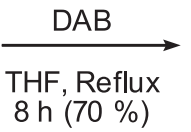
8 h $(70 \%)$ 1<smiles>CC(NCCCNS(=O)(=O)c1ccccc1[N+](=O)[O-])=C1C(=O)N(C)C(=O)N(C)C1=O</smiles>
$\mathrm{Br}\left(\mathrm{CH}_{2}\right)_{4} \mathrm{NPht}$ $\mathrm{CH}_{3} \mathrm{CN}, \mathrm{K}_{2} \mathrm{CO}_{3}$ Reflux, $20 \mathrm{~h}$ $(88 \%)$<smiles>CC(NCCCN(CCCCNc1ccccc1)S(=O)(=O)c1ccccc1[N+](=O)[O-])=C1C(=O)N(C)C(=O)N(C)C1=O</smiles>

Scheme 1.

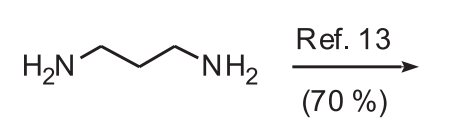<smiles>CC(C)(C)OC(=O)NCCCN</smiles>

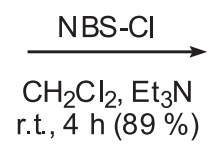

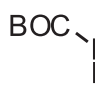<smiles>NCCCN(CCCCNc1ccccc1)S(=O)(=O)c1ccccc1[N+](=O)[O-]</smiles>

Scheme 2. 


\section{Experimental}

Melting points were determined with a Thomas-Hoover apparatus and are uncorrected. ${ }^{1} \mathrm{H}$ and ${ }^{13} \mathrm{C}-\mathrm{NMR}$ were recorded on Brucker AC 200 spetrometer. Infrared spectra were obtained with a Nicolet-550 Magna spectrophotometer. The mass spectra (MS) were obtained by electron impact $(70 \mathrm{eV})$ with a GC/VG Micromass 12 spectrometer.

The reactions were monitored by TLC analyses, on 2.0 $\mathrm{cm} \times 6.0 \mathrm{~cm}$ aluminium sheets precoated with silica gel 60 (HF-254, Merck) to a thickness of $0.25 \mathrm{~mm}$, using an ultraviolet light for visualization. For column chromatography, Merck silica gel (230-400 mesh) was used. Solvents used in the reactions were generally redistilled prior to use. The 2-nitrobenzenesulfonamides and tertbutoxycarbonyl-aminopropylamine were prepared as described for Amssoms ${ }^{8}$ and Boturyn, ${ }^{16}$ respectively.

Synthesis of (tert-butoxy)-N-(3-\{[(2-nitrophenyl)sulfonyl] aminolpropyl)carboxamide 4

To a solution of tert-butoxycarbonyl-aminopropylamine $(1.06 \mathrm{~g}, 6.08 \mathrm{mmol})$ in $\mathrm{CH}_{2} \mathrm{Cl}_{2}(20 \mathrm{~mL})$ and $\mathrm{Et}_{3} \mathrm{~N}(0.85 \mathrm{~mL})$ was added 2-nitrobenzenesulfonyl chloride $(1.35 \mathrm{~g}, 6.10$ $\mathrm{mmol}$ ). The mixture was stirred at room temperature for $4 \mathrm{~h}$. Then, the solvent was removed under reduced pressure and the oil obtained was dissolved in EtOAc and washed with brine $(3 \times 20 \mathrm{~mL})$. The organic layer was dried over anhydrous $\mathrm{Na}_{2} \mathrm{SO}_{4}$. The solvent was removed under reduced pressure, furnishing a crude solid that after purification by flash chromatography (hexane/EtOAc 10\%) yielded $1.94 \mathrm{~g}$ $(89 \%)$ of the compound 4 , as a yellow solid. mp. $97-99^{\circ} \mathrm{C}$; $\mathrm{R}_{f}=0.55$ (EtOAc/hexane 50\%); IR (KBr) $v_{\max } / \mathrm{cm}^{-1}: 3316$, 2980, 2936, 1679, 1538, 1371, 1171; ' ${ }^{1} \mathrm{H}-\mathrm{NMR}(200 \mathrm{MHz}$, $\left.\mathrm{CDCl}_{3}\right): \delta 1.41$ (s, 9H), $1.69(\mathrm{~m}, 2 \mathrm{H}, J 6.3 \mathrm{~Hz}), 3.15(\mathrm{~m}, 4 \mathrm{H})$, $4.72(\mathrm{~s}, 1 \mathrm{H}), 5.95(\mathrm{~s}, 1 \mathrm{H}), 7.74(\mathrm{~m}, 2 \mathrm{H}), 7.84(\mathrm{~m}, 1 \mathrm{H}), 8.12(\mathrm{~m}$, $1 \mathrm{H}$ ); ${ }^{13} \mathrm{C}-\mathrm{NMR}\left(50 \mathrm{MHz}, \mathrm{CDCl}_{3}\right.$ ) $\delta 28.5,30.7,37.4,41.0$, 79.8, 125.4, 131.0, 132.8, 133.7, 134.1, 148.2, 156.6; MS $\mathrm{m} / \mathrm{z}$ (rel. int.): $359\left(\mathrm{M}^{+}, 16\right), 281$ (2), 207 (5), 186 (15), 175 (100), 147 (24), 92 (6), 77(9).

Synthesis of (tert-butoxy)- $N$-(3-\{[4-(1,3-dioxoisoindolin2-yl)butyl][(2-nitrophenyl) sulfonyl]amino\} propyl) carboxamide 5

To a solution of $4(0.37 \mathrm{~g}, 1.03 \mathrm{mmol})$ in $\mathrm{CH}_{3} \mathrm{CN}(10$ $\mathrm{mL})$ was added $\mathrm{K}_{2} \mathrm{CO}_{3}(0.38 \mathrm{~g}, 3.81 \mathrm{mmol})$ and $\mathrm{N}-(4-$ bromobutyl)phthalimide $(0.29 \mathrm{~g}, 1.03 \mathrm{mmol})$. The reaction was stirred at reflux for $12 \mathrm{~h}$, after which time the solvent was removed at reduced pressure. The residue obtained was poured into water and extracted with $\mathrm{CH}_{2} \mathrm{Cl}_{2}(3 \times 10$
$\mathrm{mL})$. The combined organic layer was dried over anhydrous $\mathrm{Na}_{2} \mathrm{SO}_{4}$, filtered and concentrated under reduced pressure. The crude product was purified by flash chromatography (EtOAc/hexane $10-50 \%$ ), affording $0.42 \mathrm{~g}(71 \%)$ of the spermidine $\mathbf{5}$ as an oil. $\mathrm{R}_{f}=0.40$ (EtOAc/hexane 50\%); IR (KBr) $v_{\max } / \mathrm{cm}^{-1}: 3359,2929,2936,1770,1707,1545$, $1162 ;{ }^{1} \mathrm{H}-\mathrm{NMR}\left(200 \mathrm{MHz}, \mathrm{CDCl}_{3}\right): \delta 1.42(\mathrm{~s}, 9 \mathrm{H}), 1.60(\mathrm{~m}$, $4 \mathrm{H}), 1.73(\mathrm{~m}, 2 \mathrm{H}), 3.14(\mathrm{~m}, 2 \mathrm{H}), 3.34(\mathrm{~m}, 4 \mathrm{H}), 3.64(\mathrm{t}, 2 \mathrm{H}, J$ $6.3 \mathrm{~Hz}), 4.90(\mathrm{~s}, 1 \mathrm{H}), 7.63-7.69(\mathrm{~m}, 2 \mathrm{H}), 7.72(\mathrm{~m}, 2 \mathrm{H}) 7.83$ $(\mathrm{m}, 1 \mathrm{H}), 8.00(\mathrm{~m}, 1 \mathrm{H}) ;{ }^{13} \mathrm{C}-\mathrm{NMR}\left(50 \mathrm{MHz}, \mathrm{CDCl}_{3}\right) \delta 25.5$, 25.8, 28.7, 28.7, 45.3, 47.1, 79.3, 123.4, 124.3, 130.7, 131.8, 132.1, 133.5, 133.6, 134.1, 148.1, 156.1, 168.4.

Synthesis of 1,3-dimethyl-5-\{[(3-\{[(2-nitrophenyl) sulfonyl]amino ? propyl)amino] ethylidene $\}-1,3$ dihydropyrimidine-2,4,6-trione 1

To a solution of 5-(1-hydroxyethylidene)-1,3dimethylhexahydro-2,4,6-pyrimidinetrione (DAB, 0.101 $\mathrm{g}, 0.510 \mathrm{mmol})$ in THF $(10 \mathrm{~mL})$ was added 1 equivalent of 2-nitrobenzenesulfonamidopropylamine $(0.114 \mathrm{~g} ; 0.510$ $\mathrm{mmol})$. The mixture was stirred at reflux for $8 \mathrm{~h}$. After the reaction was judged complete, the solvent was removed under reduced pressure to afford a solid that after purification by flash chromatography $\left(\mathrm{SiO}_{2}, 230-400\right.$ mesh, EtOAc/hexane 50-70\%), yielded $0.157 \mathrm{~g}(70 \%)$ of 1 as a white solid. m.p. $122-124{ }^{\circ} \mathrm{CR}_{f}=0.12$ (AcOEt/hexane $50 \%)$; IR (KBr) $v_{\max } / \mathrm{cm}^{-1}: 3591,3353,3092,2947,1705$, $1645,1589,1480,1356,1156,855,754,598,426 ;{ }^{1} \mathrm{H}-$ NMR (CDCl, $200 \mathrm{MHz}): \delta 12.62(\mathrm{~s}, 1 \mathrm{H}), 7.25-8.18(\mathrm{~m}$, $4 \mathrm{H}), 5.65(\mathrm{~s}, 1 \mathrm{H}), 3.58(\mathrm{q}, 2 \mathrm{H}, J 6.28 \mathrm{~Hz}), 3.30(\mathrm{~s}, 6 \mathrm{H}), 3.24$ (q, $2 \mathrm{H}, J 6.28 \mathrm{~Hz}$ ), 2.68 (s, 3H), 1.98 (quint, $2 \mathrm{H}, J 6.28 \mathrm{~Hz}$ ), ${ }^{13} \mathrm{C}-\mathrm{NMR}\left(\mathrm{CDCl}_{3}, 50 \mathrm{MHz}\right): \delta 18.0,28.0,29.7,40.8,90.7$, 125.6, 131.3, 133.0, 133.4, 134.0, 148.2, 151.5, 163.0, 166.6, 174.6; MS $m / z$ (rel. int.): 439 ( $\left.\mathrm{M}^{+} 17\right), 422$ (13), 224 (100), 210 (42), 197 (22), 181 (24), 56 (18). HRMS calcd for $\mathrm{C}_{17} \mathrm{H}_{21} \mathrm{~N}_{5} \mathrm{O}_{7} \mathrm{~S}: 439.1161$. Found: 439.1158

Synthesis of 5-\{[(3-\{[4-(1,3-dioxoisoindolin-2-yl) butyl][(2-nitrophenyl)sulfonyl] amino\} propyl) amino Jethylidene \}-1,3-dimethyl-1,3-dihidropyrimidine2,4,6-trione 2

To a solution of $\mathbf{1}(0.15 \mathrm{~g}, 0.34 \mathrm{mmol})$ in $\mathrm{CH}_{3} \mathrm{CN}$ (20 $\mathrm{mL}$ ) were added $\mathrm{K}_{2} \mathrm{CO}_{3}(0.10 \mathrm{~g}, 1.02 \mathrm{mmol})$ of potassium carbonate, N-(4-bromobutyl)phthalimide $(0.96 \mathrm{~g}, 0.34$ $\mathrm{mmol}$ ) and a few crystals of KI. The reaction was stirred at reflux for $20 \mathrm{~h}$. Then, solvent was removed at reduced pressure and the residue obtained was poured into water and extracted with $\mathrm{CH}_{2} \mathrm{Cl}_{2}(3 \mathrm{X} 10 \mathrm{~mL})$. The combined organic layer was dried over anhydrous $\mathrm{Na}_{2} \mathrm{SO}_{4}$, filtered 
and concentrated under reduced pressure, furnishing a crude solid that after purification by flash chromatography ( $\mathrm{MeOH} / \mathrm{CH}_{2} \mathrm{Cl}_{2} 5 \%$ ), yielded $0.191 \mathrm{~g}(88 \%)$ of spermidine 2 as a white solid. m.p. $68-70{ }^{\circ} \mathrm{C} ; \mathrm{R}_{f}=0.55(\mathrm{MeOH} /$ $\mathrm{CH}_{2} \mathrm{Cl}_{2} 5 \%$ ); IR (KBr) $v_{\max } / \mathrm{cm}^{-1}: 3462,2925,2854,1770$, 1711, 1640, 1593, 1543, 1477, 1161; ${ }^{1} \mathrm{H}-\mathrm{NMR}(200 \mathrm{MHz}$, $\left.\mathrm{CDCl}_{3}\right): \delta 1.63(\mathrm{~m}, 4 \mathrm{H}), 1.99(\mathrm{q}, 2 \mathrm{H}, J 7.14 \mathrm{~Hz}), 2.65$ (s, $3 \mathrm{H}), 3.29(\mathrm{~s}, 6 \mathrm{H}), 3.44(\mathrm{~m}, 6 \mathrm{H}), 3.65(\mathrm{t}, 2 \mathrm{H}, J 6.06 \mathrm{~Hz}), 7.60-$ $7.73(\mathrm{~m}, 7 \mathrm{H}), 8.01(\mathrm{~m}, 1 \mathrm{H}), 12.60(\mathrm{~s}, 1 \mathrm{H}) ;{ }^{13} \mathrm{C}-\mathrm{NMR}(50$ $\left.\mathrm{MHz}, \mathrm{CDCl}_{3}\right) \delta 18.0,25.4,25.8,27.9,28.2,28.2,37.1$, 41.2, 44.9, 47.3, 90.7, 123.3, 124.3, 131.1, 131.8, 132.1, $133.1,133.8,134.1,148.1,163.0,166.68,168.4,174.5$.

Synthesis of 2-(4-\{(3-aminopropyl)[(2-nitrophenyl) sulfonyl]amino (butyl)isoindoline-1,3-dione 3

To a solution of spermidine $2(0.20 \mathrm{~g} ; 0.31 \mathrm{mmol})$ in THF $(15 \mathrm{~mL})$ at $0{ }^{\circ} \mathrm{C}$ was added 2 eq. of hydrazine hidrate $(0.62 \mathrm{mmol}, 0.04 \mathrm{~mL})$. The reaction was stirred at $0{ }^{\circ} \mathrm{C}$ for $3.5 \mathrm{~h}$. Then, the solution was filtered on a short pad of silica gel ( $\left.\mathrm{MeOH} / \mathrm{Et}_{3} \mathrm{~N} 50 \%\right)$ and the solvent was removed under reduced pressure. The resulting product was purified by flash chromatography (EtOAc and then $\mathrm{MeOH} / \mathrm{Et}_{3} \mathrm{~N}$ $50 \%$ ). The spermidine 3 was obtained as an oil in $83 \%$ yield (unstable). $\mathrm{R}_{f}=0.65\left(\mathrm{MeOH} / \mathrm{Et}_{3} \mathrm{~N} 50 \%\right)$ IR (film) $v_{\max } / \mathrm{cm}^{-1}: 3450,2930,2854,1711,1593,1543,1477$, 1161; ${ }^{1} \mathrm{H}-\mathrm{NMR}\left(200 \mathrm{MHz}, \mathrm{CDCl}_{3}\right): \delta$ 1.47-1.85 (m, 6H), $2.41(\mathrm{~m}, 4 \mathrm{H}), 2.79(\mathrm{~s}, 2 \mathrm{H}), 3.58(\mathrm{t}, 2 \mathrm{H}, J 6.91 \mathrm{~Hz}), 7.55-7.75$ $(\mathrm{m}, 7 \mathrm{H}), 8.05(\mathrm{~m}, 1 \mathrm{H}){ }^{13} \mathrm{C}-\mathrm{NMR}\left(50 \mathrm{MHz}, \mathrm{CDCl}_{3}\right) \quad \delta$ 23.4, 26.4, 37.8, 39.6, 58.5, 52.0, 52.9, 123.3, 124.3, 131.1, $131.9,132.2,133.1,133.9,134.0,148.2,168.5$.

\section{Electronic Supplementary Information}

${ }^{1} \mathrm{H}$ and ${ }^{13} \mathrm{C}$ NMR spectra for compounds $2,3,4$ and 5 available as PDF file at http://jbcs.sbq.org.br

\section{Acknowledgements}

The authors would like to thank the European Community, International Foundation for Sciences and FAPERJ for financial support. E.T.S. would like to thank CAPES and FAPERJ for a fellowship. We also thank the Laboratory of Mass Spectrometry of the Institute of Chemistry of State University of Campinas.

\section{References}

1. Bacchi, C.J.; Lane, S.; Weiss, L.M.; Yarlett, N.; Takvorian, P.; Wittner, M.; J. Eukaryot. Microbiol. 2001, 48, 374.
2. Zhang, X.; Rao, M.N.; Jones, S. R.; Shao, B.; Feibush, P.; McGuigan, M.; Tzodikov, N.; Feibush, B.; Sharkansky, I.; Snyder, B.; Mallis, L.M.; Sarkahian, A.; Wilder, S.; Joshua, E.T.; Kinney, W. A.; J. Org. Chem. 1998, 63, 8599; Bergeron, R.J.; Muller, R.; Huang, G.F.; McManis, J.S.; Algee, S.E.; Yao, H.; Weimar, W.R.; Wiegand, J.; J. Med. Chem. 2001, 44, 2451; Hone, N.D.; Payne, L.J.; Tetrahedron Lett. 2000, 41, 6149.

3. Gonzlez, N.S.; Huber, A.; Alagranati, I.D.; FEBS Letters 2001, 508, 323.

4. Muller, S.; Coombs, G.H.; Walter; R.D.; Trends Parasitol. 2001, 17, 242.

5. Olliaro, P.; Lazdins, J.; Guhl, F.; Expert Opin. Emerging Drugs 2002, 7, 1 .

6. Schirmer, R.H.; Müller, J.G.; Krauth-Siegel, R.L.; Angew. Chem. Int. Ed. 1995, 34, 141.

7. D’Silva, C.; Daunes, S.; Expert Opin. Ivestig. Drugs 2002, 11, 217; Krauth-Siegel, R.L.; Coombs, G.H.; Parasitol. Today 1999, $15,404$.

8. Amssoms, K.; Oza, S.L.; Augustyns, K.; Yamani, A.; Lambeir, A.-M.; Bal, G.; Van der Veken, P.; Fairlamb, A.H.; Haemers, A.; Bioorg. Med. Chem. Lett. 2002, 12, 2703; Amssoms, K.; Oza, S.L.; Ravaschino, E.; Yamani, A.; Lambeir, A.-M.; Rajan, P.; Bal, G.; Rodriguez, J.B.; Fairlamb, A.H.; Augustyns, K.; Haemers, A.; Bioorg. Med. Chem. Lett. 2002, 12, 2553; Chibale, K.; Chipeleme, A.; Warren, S.; Tetrahedron Lett. 2002, 43, 1587; Augustyns, K.; Amssoms, K.; Yamani, A.; Rajan, P.K.; Haemers, A.; Curr. Pharm. Design 2001, 7, 1117; Garrard, E.A.; Borman, E.C.; Cook, B.N.; Pike, E.J.; Alberg, D.G.; Org. Lett. 2000, 2, 3639; Chen, S.; Lin, C-H.; Walsh, C.T.; Coward, J.K.; Bioorg. Med. Chem. Lett. 1997, 5, 505.

9. Silva, E.T.; Cunha, A.S.; Lima, E.L.S.; Bioorg. Med. Chem. Lett. 2002, 12, 3207.

10. Kuksa, V.; Buchan, R.; Lin, P.K.T.; Synthesis 2000, 1189; Karigiannis, G.; Papaioannow, D.; Eur. J. Org. Chem. 2000, 1841.

11. Fukuyama, T.; Jow, C.-K.; Cheung, M.; Tetrahedron Lett. 1995, 36, 6373.

12. Nihei, K.; Kato, M. J.; Yamane, T.; Palma, M.S.; Konno, K.; Bioorg. Med. Chem. Lett. 2002, 12, 299.

13. Amssoms, K.; Augustyns, K.; Yamani, A.; Zhang, M.; Haemers, A.; Synth. Commun. 2002, 32, 319.

14. da Silva, E.T.; Lima, E.L.S.; Tetrahedron Lett. 2003, 44, 3621.

15. Saab, N.H.; West, E.E.; Bieszk, N.C.; Preuss, C.V.; Mank, A.R.; Casero, R.A.; Woster, P.M.; J. Med. Chem. 1993, 36, 2998.

16. Boturyn, D.; Boudali, A.; Constant, J.F.; Defrancq, E.; Lhomme, J.; Tetrahedron 1997, 53, 5485.

Received: April 4, 2003 Published on the web: April 30, 2004 